\title{
Scenario Technology for Planning C2 Exercises
}

\author{
Carla Burns \\ Air Force Research Laboratory \\ 525 Brooks Rd \\ Rome NY 13441-4505 \\ (315) 330-2638 \\ burnsc@rl.af.mil
}

\begin{abstract}
Over the years, military exercises have proven to increase our country's effectiveness in actual missions. However, the exercise planning process is a time consuming activity that can span anywhere from months to years. Scenarios are often used during C2 exercise planning and execution to portray training tasks in the context of real-world situations. Exercise scenarios typically originate as a few high level paragraphs or pages of textual and/or graphical information and evolve into very detailed events as the exercise planning process progresses. The development of a detailed scenario to support an exercise is a time consuming process that often accounts for a large portion of the exercise planning cycle. Thus, there is a need for technology that enhances the exercise planning process by shortening the amount of time it takes to create and validate detailed exercise scenarios against training tasks and available resources. This paper discusses the capabilities of the Air Defense Exercise Planning Tool (ADEPT). The goal of the ADEPT technology is to shorten the exercise planning cycle by providing automated scenario development, analysis and generation capabilities. In addition, the paper addresses current limitations of existing scenario technology, the results of small pilot project and future capabilities for ADEPT.
\end{abstract}

\section{Introduction}

As we move into the $21^{\text {st }}$ century, modern warfare will embody improved intelligence and command and control usage by joint forces. Joint Vision 2010 is a document that provides a conceptual framework for how United States forces will leverage technological opportunities to achieve new levels of effectiveness in joint warfighting [Joint Chiefs of Staff, 1998]. Joint Vision 2010 states that continued mission rehearsal and training are critical to meeting future military objectives. Over the years, evaluated training whether it be in the form of simulation, full blown exercises, or joint coalition training have increased our effectiveness. This training must be sustained to allow our forces to be ready for the diverse types of missions they will encounter in the $21^{\text {st }}$ century. At the same time, $21^{\text {st }}$ century forces will strive to dynamically plan and execute missions with fewer personnel over a shorter duration. Thus, there is a need for technology that enhances the exercise planning process by shortening its planning cycle via automated exercise development, analysis and generation capabilities. 


\section{Scenarios and Their Role in the Exercise Planning Process}

Exercise planning cycles can span anywhere from months to years. The joint exercise planning process begins by determining the tasks the forces will train against given task priorities and available resources. These essential tasks are referred to as the Joint Mission Essential Task List (JMETL) [Joint Chief of Staff, 1995]. The assigned mission, its plan, and joint doctrine are among the factors considered when developing the JMETL. Scenarios are often used during the planning and execution of exercises to portray the JMETL tasks in the context of a real-world situation. In addition, scenarios explore the various options for given situations and scope the training based on the exercise resources available.

Exercise scenarios typically originate as a few high level paragraphs or pages of textual and/or graphical information and evolve into very detailed events as the exercise planning process progresses. Such detailed scenarios are often referred to as the Master Sequence of Events List (MSEL). A MSEL provides a specific event(s) at a given time in the exercise along with other informative data. A typical exercise often contains hundreds or thousands of MSELs. The development of such detailed scenarios or MSELs to support an exercise is a time consuming process that often accounts for a large portion of the exercise planning cycle. Figure 1 provides a graphical depiction of how scenarios evolve during the exercise planning process.

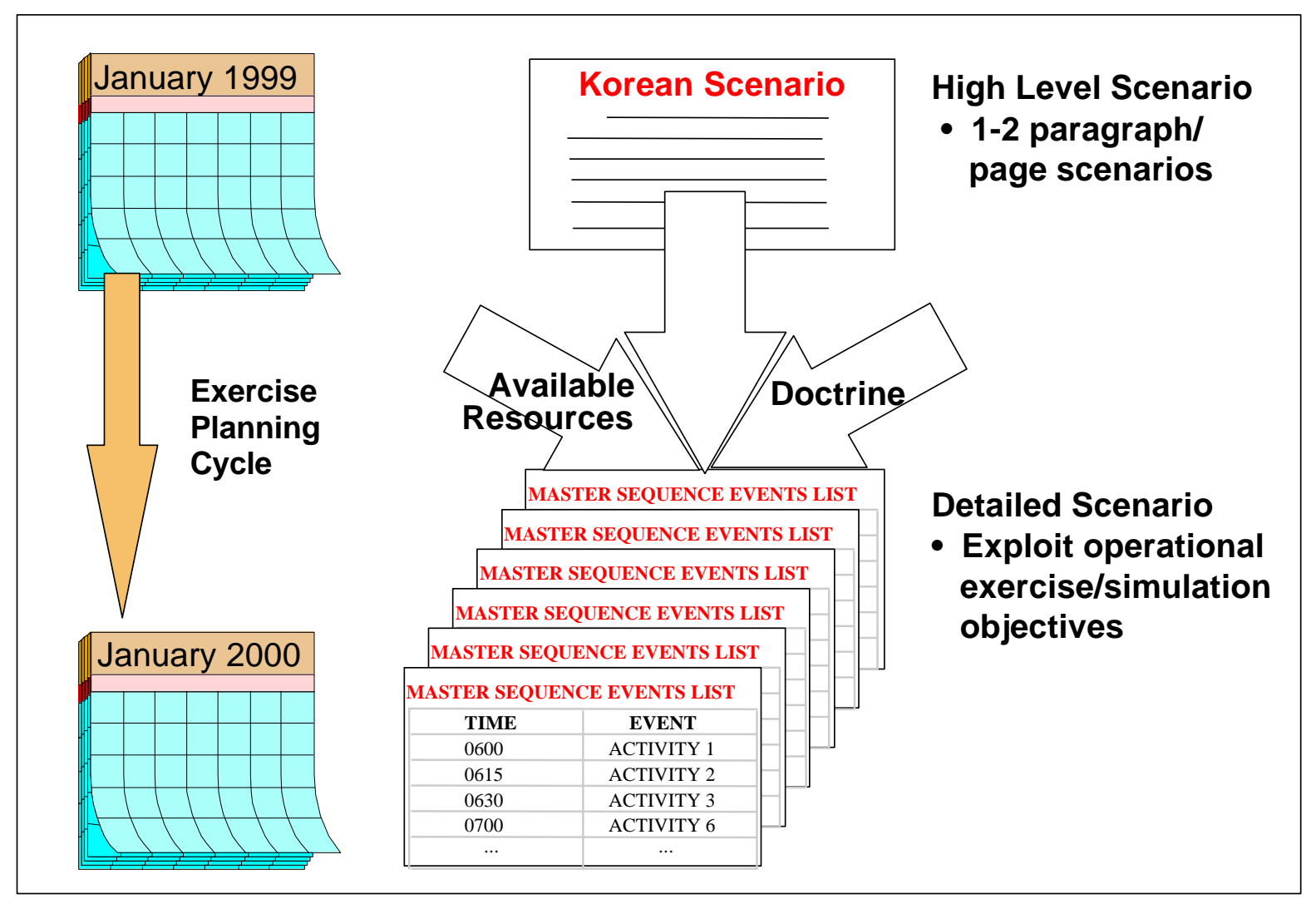

Figure 1. Scenario Evolution During the Exercise Planning Process. 


\subsection{Current Technology Limitations}

There are several limitations with existing technology that is used to construct detailed scenarios or MSELs that impact the efficiency of the exercise planning process. First, current MSEL tools provide non-graphical, clumsy user interfaces to input the massive amounts of data required for an exercise. Second, tools often fail to provide mechanisms for reusing detailed scenarios or MSELs and their components. It is often the case that exercises utilize the same types of events or MSELs over and over but vary the data instances to support different circumstances. Examples of event types include a terrorist attack, an unknown track entering airspace, or a security incident. Third, the tools fail to provide traceability from exercise goals to events in the detailed scenarios or MSELs. This capability allows one to more easily identify which training tasks are not covered in the scenario prior to exercise execution or simulation. Large, joint exercises could greatly benefit from such traceability mechanisms. Finally, other than checking for invalid inputs or spelling, today's technology fails to provide automated MSEL analysis or generation capabilities.

\subsection{The Air Defense Exercise Planning Tool (ADEPT)}

Air Force Research Laboratory (AFRL) has developed technology to support detailed scenario or MSEL development that is aimed at shortening the exercise planning cycle. The Air Defense Exercise Planning Tool (ADEPT) prototype provides a drag and drop, mixed media approach for MSEL construction. It supports case-based MSEL development by providing facilities for reusing existing MSEL components to construct new exercises and enhance existing ones. Traceability from scenario events to joint doctrine is supported. The ultimate goal of the AFRL scenario program is unification of the exercise planning products to support automated analysis and generation of skeleton scenarios or MSELs. An underlying scenario representation model is the key aspect of the approach to meeting these objectives. ADEPT is compatible with the Joint Exercise Management Program (JEMP) MSEL construction tool. The ADEPT software runs on a PC/NT or Windows platform and leverages the COTS Microsoft Office product.

\subsection{MSEL Construction Using ADEPT}

An illustrative exercise example containing numerous MSELs captured in ADEPT is depicted in Figure 2. The ADEPT screen is comprised of two primary regions. The left region provides a listing of available events for potential use in an exercise. The right area represents an actual exercise where events are specified to occur at a given time for a specified duration.

\subsubsection{Defining Available Exercise Events}

Exercise events are created using one of three options. Users may import events or MSELs from either the JEMP tool or other ADEPT exercises. An import exercise operation places the entire contents of the import file into the exercise events list and the exercise workspace. Thus, the MSEL events become part of the actual exercise and contain all of the associated event data that the user can then tailor to meet his/her needs. Figure 3 illustrates all of the event data imported into an exercise for a message event that indicates a $\mathrm{C}-130$ has crashed and requires assistance. 


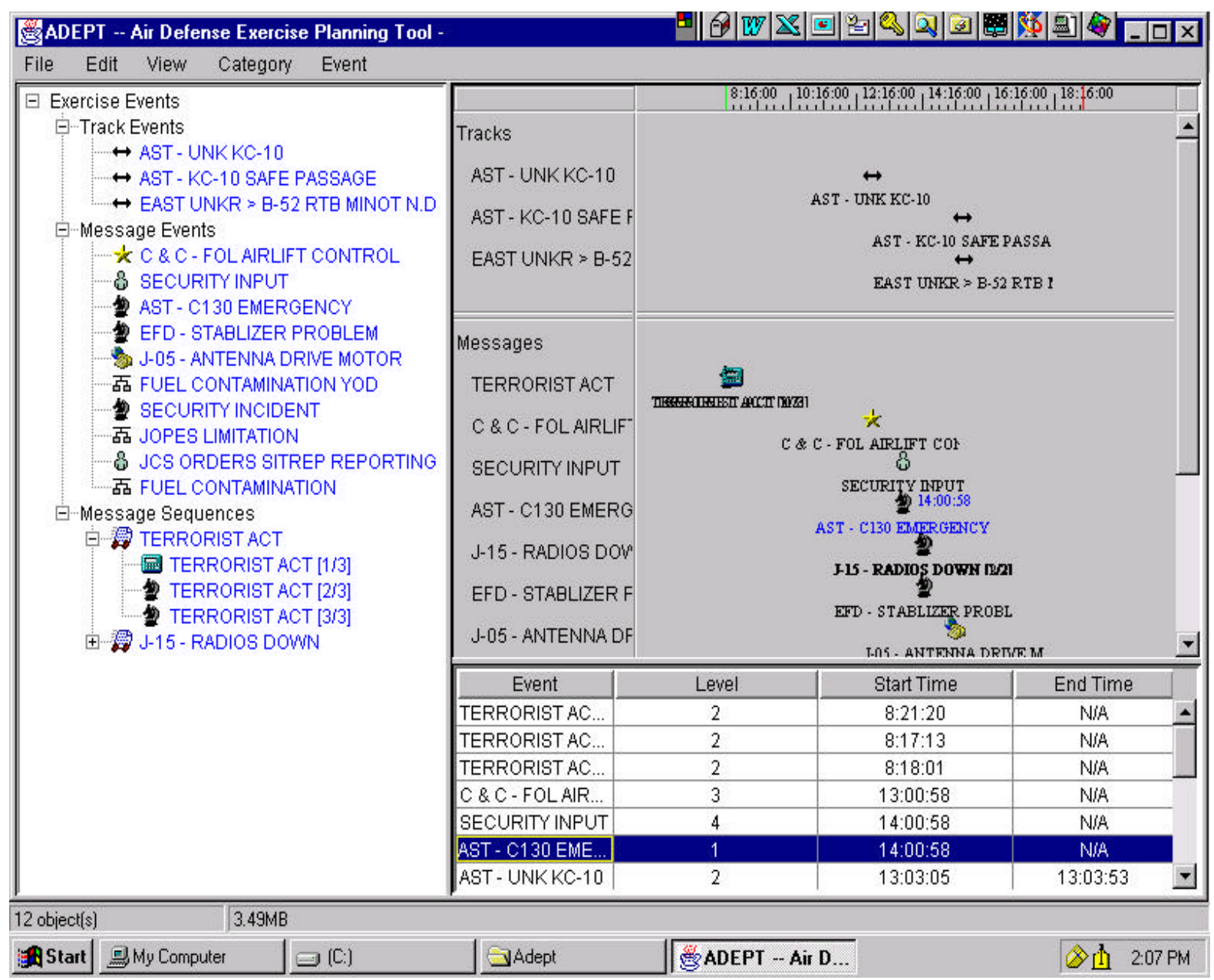

Figure 2. An Exercise Represented in ADEPT.

Exercise events can also be made available by extracting them from a library. Contrary to the import exercise operation, importing an event from a library places the event and its associated data in the exercise event list - NOT in the actual exercise workspace. The user makes events part of an exercise by selecting them from the available event list and dragging them over into the Exercise Workspace. Events in the list that are not part of the exercise are colored coded for the user. Finally, exercise events can be created from scratch.

ADEPT classifies all events as one of three types: tracks, messages and message sequences. Track events represent aircraft events. Message events are used for communication activities. Message sequences are normal patterns of messages that typically occur in a given situation. An example of a message sequence would be an equipment failure. The sequence of messages would indicate that a piece of equipment has failed, provide an estimated time for when it might become operational, and notify when the equipment has been fixed. 


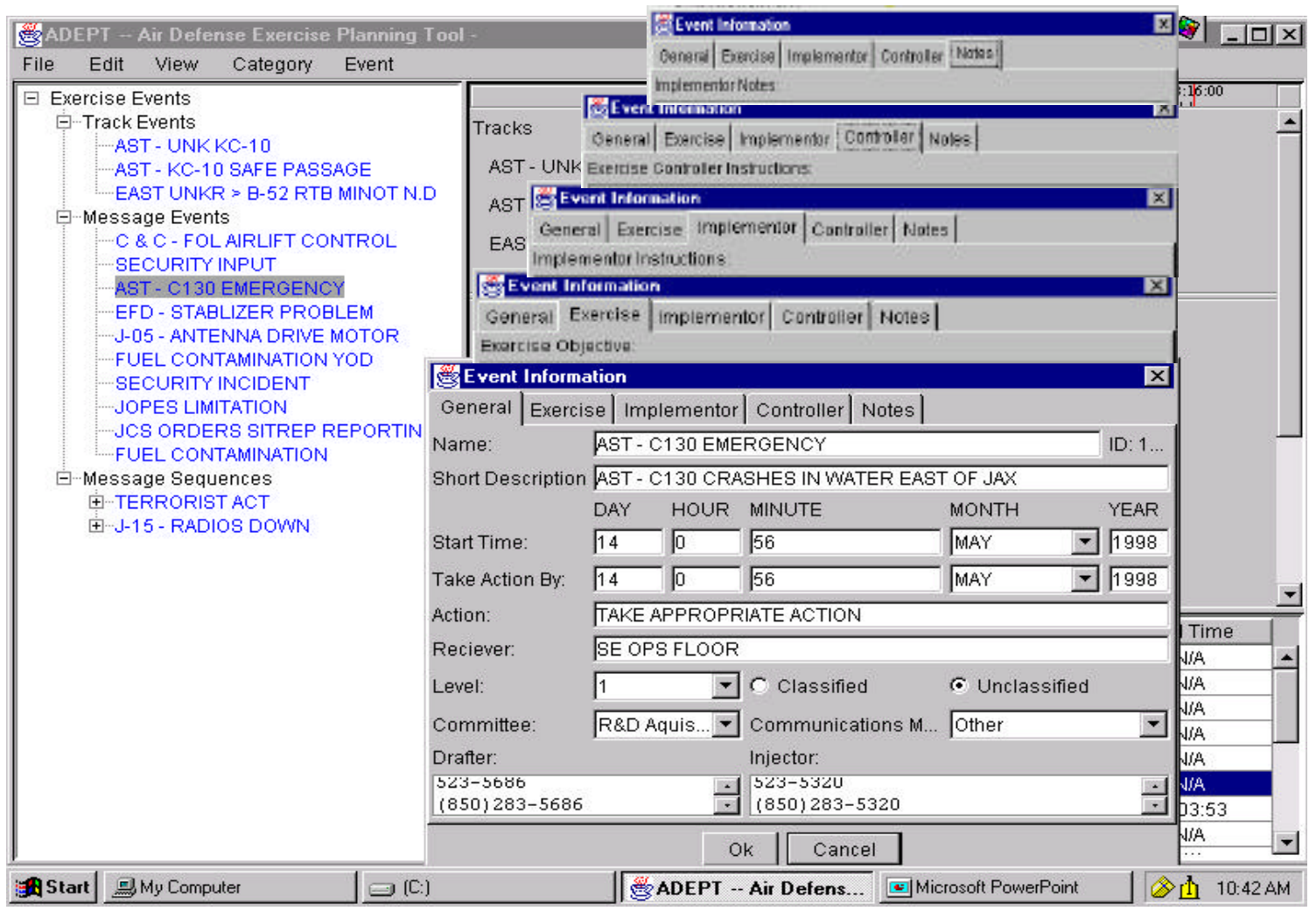

Figure 3. Event Data Imported into an Exercise.

\subsubsection{Populating an Exercise}

Exercises are built and/or enhanced using the available exercise event list whereby events can be directly imported into an exercise, selected and moved over into the workspace or defined from scratch. An exercise timeline that reflects the length of the exercise is displayed across the top of the exercise workspace. Specific times for exercise events can be defined and modified simply by moving an event to the desired location on the timeline. Zoom in and zoom out capabilities are provided to better support viewing data on the timeline. In addition, a big picture exercise view that contains all of the exercise events is supported. The ADEPT exercise workspace also provides consistent timeline and table views of exercise data as illustrated in Figure 2. Thus, the user has the choice of using graphical and/or tabular means to build exercises.

One of the drawbacks of current record-based MSEL tools used to capture exercise events is that they fail to provide organizational structures to support filtered views of exercise data. For example, users may wish to organize an exercise around all of the events affiliated with a geographical location, mission function or $\mathrm{J}$ group. The ADEPT tool provides logical grouping mechanisms called categories to support the grouping of events. Figure 4 portrays an exercise that partitions events based on the geographic locations of Langley Air Force Base, Hurlburt Field, and Fort Drum as indicated on the left side of the screen. Such logical grouping mechanisms can greatly assist the management of large, joint exercises to help insure that everyone involved knows who is working on what exercise event with whom, when in the exercise. 


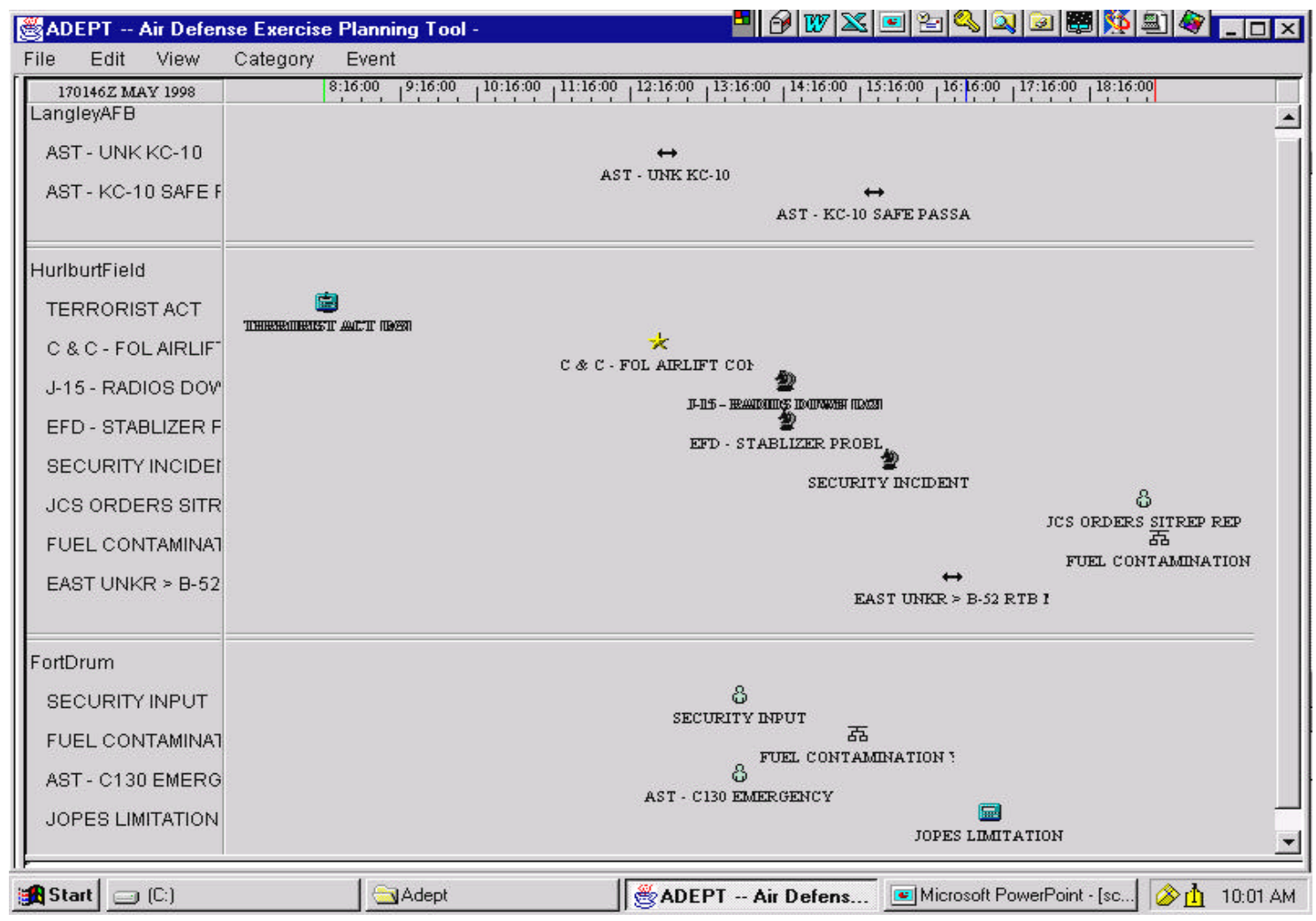

Figure 4. Illustration of Event Grouping Mechanisms in ADEPT.

\subsection{Relating Exercise Events to Doctrine}

Another useful tool for planning large, joint exercises is that of providing traceability from mission objectives (JMETL) to those events in the detailed scenarios (MSELs) which satisfy them. This allows one to more easily determine which training tasks in the JMETL are not covered in the exercise scenario prior to the exercise execution or simulation. In addition, one can more readily keep track of tasks that have and have not been trained against over time. Figure 5 illustrates how ADEPT graphically supports the mapping of exercise events to the Joint Mission Essential Tasks. The program's approach of coalescing the exercise planning products (e.g. JMETL, MSEL, Joint Lessons Learned (JULL)) also provides the underlying data framework for automated exercise analysis capabilities. Such analysis capabilities could include the discovery of unmet training objectives based on their absence from detailed scenarios and the identification of timing conflicts within a scenario. 


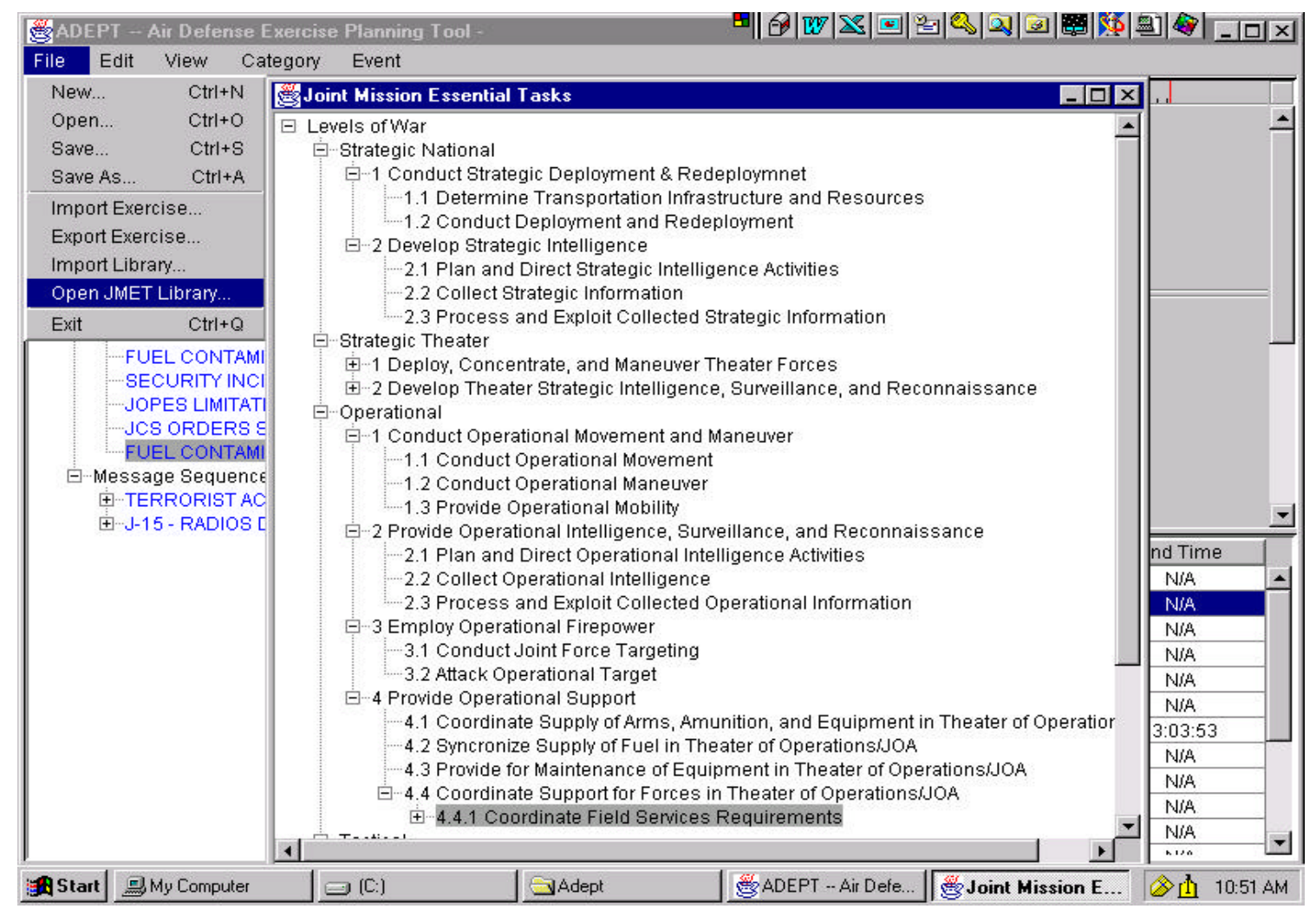

Figure 5 - Relating Exercise Events to Doctrine using ADEPT.

\subsection{Scenario Representation Model}

The ultimate goal of the AFRL scenario program is unification of the exercise planning products to support automated analysis and generation of skeleton scenarios or MSELs. An underlying scenario reference model serves as the heart of the ADEPT tool to support these goals. The scenario reference model merges concepts from three prevalent scenario methods: the Use Case as defined by Jacobsen [Jacobsen, 1992]; a Task Script as defined by Graham [Graham, 1995]; and the Scenario Schematic as defined by Potts [Potts, 1995]. Figure 6 provides a graphical depiction of the scenario reference model that defines the objects and attributes used to represent scenario information in ADEPT. It is important to note that one may still individually capture any of the three methods identified above in the scenario reference model.

Each detailed scenario in ADEPT is related to goals or objectives and other scenarios. Components in the model represent entities such as scenario context, initial conditions, measurement attributes, roles and actors, importance criteria, and clarification data items. There is also the capability to relate objects in the model to data dictionaries or conceptual models that ensure the objects are defined within a given domain. A set of Java classes and a relational database schema has been created to represent the semantics of the model. The underlying scenario reference model has been applied across the following domains to validate the relationships within the model and address scalability: exercise planning, modeling \& simulation, computer based training, and systems engineering. 


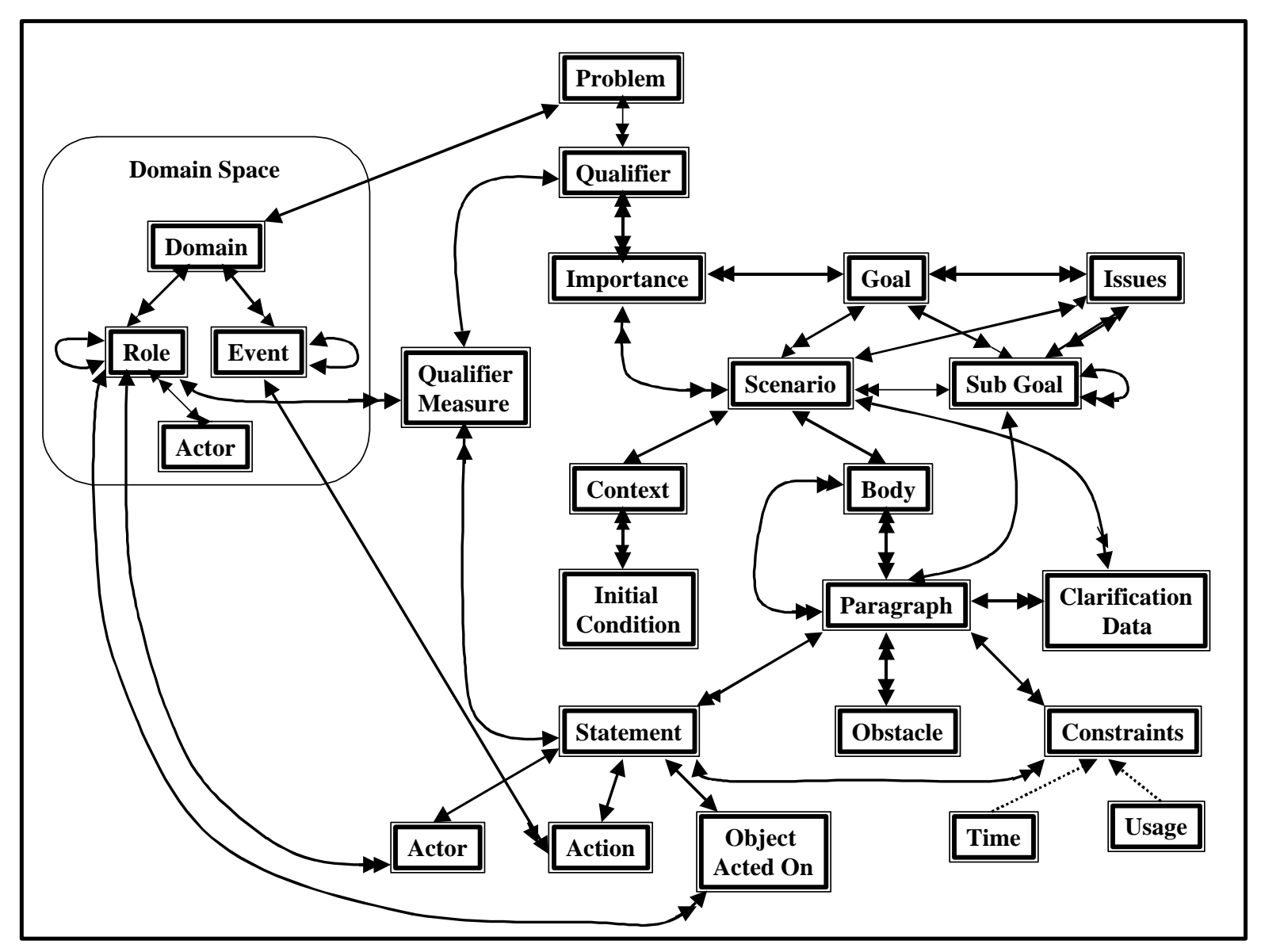

Figure 6. The Scenario Reference Model.

\subsection{Results of ADEPT Pilot Project}

A small pilot project was conducted using the ADEPT prototype. The application focused on a Northeast Defense Sector (NEADS) portion of a NORAD scenario. It is estimated that NEADS use of ADEPT on the same NORAD scenario would result in a productivity improvement factor of eight over the use of their existing MSEL technology. The MSEL reuse and graphical definition capabilities of ADEPT serve as the basis for the results.

\subsection{Future ADEPT Capabilities}

The ADEPT prototype currently helps reduce the exercise planning process by shortening the amount of time it takes to generate detailed exercise scenarios or MSELs using a graphical and case-based approach to MSEL development. Future work will incorporate automated analysis and generation techniques by leveraging the exercise data represented in the underlying scenario reference model. Several additional capabilities have been identified for ADEPT based on working with end users. Among them include multi-user collaboration capabilities, integration of map facilities, linkage to simulation tools and big screen, dual monitor support for working with large exercises. 


\subsection{Acknowledgements}

The ADEPT prototype was developed by Modus Operandi, Inc. for Air Force Research Laboratory under Contract Number F30602-96-C-0050. The author would especially like to thank Tony Stirtzinger and Lee Krause for their hard work and contributions to the ADEPT project. In addition, many thanks to all of the end users AFRL felt very fortunate to work with including MSgt Lyle Seelbach and others from the Northeast Air Defense Sector (NEADS), Herb Bruse and the 505th Exercise Support Squadron and Maj Andy Butler of the $152^{\text {nd }}$ Air Control Group.

\subsection{References}

[Jacobsen, 1992] Jacobsen, Ivor. Object-Oriented Engineering. Addison-Wesley, New York, 1992.

[Joint Chiefs of Staff, 1998] Joint Chiefs of Staff. Joint Vision 2010, Pentagon, Washington D.C., 1998.

[Joint Chief of Staff, 1995] Joint Chiefs of Staff. Joint Mission Essential Task List (JMETL) Development Handbook. Pentagon, Washington D.C. December 1995.

[Graham, 1995] Graham, Ian. Task Scripts, Use Cases and Scenarios with Object-Oriented Analysis. Object Oriented Systems 3, 1996.

[Potts, 1995] Potts, Colin. "Using Schematic Scenarios to Understand User Needs". Symposium on Designing Interactive Systems: Processes, Practices, Methods and Techniques, August 1995. 